\title{
El Ejército Nacional de Colombia como agente de desarrollo territorial: una revisión al programa Fe en Colombia en el departamento del Caquetá
}

The Colombian National Army as an Agent of Territorial Development: A Survey of the Fe en Colombia Program in the Department of Caquetá

Paula Julieth Penagos Medina* Universidad Santo Tomás, Bogotá, Colombia http://orcid.org/0000-0001-9707-111X

Artículo de investigación

Fecha de recepción: 13 de febrero de 2020 Fecha de aceptación: 24 de abril de 2020

\section{Para citar este artículo}

Penagos Medina, P. J. (2020). El Ejército Nacional de Colombia como agente de desarrollo territorial: una revisión al programa Fe en Colombia en el departamento del Caquetá. Campos en Ciencias Sociales, 8(2), 291-311. DoI: https://doi. org/10.15332/25006681/6020

\section{RESUMEN}

El departamento del Caquetá ha sido uno de los territorios más afectados por el conflicto armado colombiano, hecho que debería ser directamente proporcional al esfuerzo institucional

Profesional Cum Laude en Gobierno y Relaciones Internacionales de la Universidad Santo Tomás, especialista en Paz y Desarrollo Territorial. Correo electrónico: paula.penagos@usantotomas.edu.co 
por la recuperación social y el desarrollo de las comunidades en el posconflicto. En ese orden de ideas, el Ejército Nacional, a través de su programa Fe en Colombia, realiza acciones con miras a fortalecer las relaciones cívico-militares e incidir en el desarrollo del departamento a través de la asunción de nuevas funciones que responden a un contexto en transformación y un concepto de seguridad ampliado. Este texto examina el rol de dicha institución en la generación de desarrollo en el Caquetá a partir de la iniciativa Fe en Colombia.

Palabras clave: Ejército Nacional de Colombia, Fe en Colombia, desarrollo.

\begin{abstract}
The Department of Caquetá has been one of the territories most affected by the Colombian armed conflict, a fact that should be directly proportional to the institutional effort for social recovery and the development of communities during the post-conflict period. In this vein, the National Army, through its $\mathrm{Fe}$ in Colombia (Faith in Colombia) Program, carries out actions with a view to strengthening civil-military relations and influencing the development of the Department by undertaking of new functions that respond to a changing context and an expanded concept of security. This text examines the role of this institution in generating development in Caquetá from the Fe en Colombia initiative.
\end{abstract}

Keywords: Colombian National Army, Fe en Colombia, development.

\title{
INTRODUCCIÓN
}

La narrativa que se ha construido del término Caquetá responde a imaginarios de violencia, conflicto armado, pobreza y guerrilla, que vienen a tener su cúspide en la relación de dicho territorio con el fallido proceso de paz del Caguán en el 2002. Por otra parte, en medio del posconflicto y con la asunción de nuevos procesos sociales y actores políticos, también ha tenido lugar la transformación paulatina de las funciones de las Fuerzas Militares de Colombia, especialmente del Ejército Nacional, con miras a convertirse en un agente de construcción de paz. 
Caquetá es uno de los seis departamentos con mayor tasa de incidencia del conflicto armado colombiano, con base en el número de acciones armadas, homicidios, secuestros, víctimas de minas antipersonal, desplazamiento forzado y cultivos de coca (Departamento Nacional de Planeación, 2016).

Si bien las estadísticas e infografías oficiales del conflicto armado muestran que el departamento más afectado por la violencia en Colombia ha sido Antioquia, también es cierto que, de acuerdo con el número de habitantes, el mayor impacto demográfico del conflicto armado lo han vivido departamentos como Caquetá. Esto se traduce, a su vez, en una desaceleración de la economía, un resquebrajamiento del tejido social y una pérdida de capital humano.

Por lo anterior, la construcción de paz implica la participación de las instituciones del Estado, incluyendo el Ejército Nacional, mediante lo que se conoce como acción integral ${ }^{l}$, que incluye al sector privado, la sociedad civil y la cooperación internacional. Ahora bien, la participación de las Fuerzas Militares, especialmente del Ejército Nacional, ha sido determinante en la conducción del conflicto armado de esta región, no solo por ser un actor de este, sino porque se ha convertido en la institución interlocutora entre las comunidades y el Gobierno Nacional.

De esta manera, a través de los años, el Ejército Nacional ha diseñado diferentes estrategias y programas para acercarse a la población, promoviendo el desarrollo y bienestar de estas, y — lo que resulta más importante- complejizando la victoria militar del adversario mediante la restauración de los vínculos de confianza de las comunidades caqueteńas con la institucionalidad.

En ese orden de ideas, Fe en Colombia es:

1 La doctrina de acción integral y desarrollo de las Fuerzas Militares se define como la acción social del Estado en la recuperación de los territorios mediante acciones coordinadas entre las instituciones estatales en aras de llevar bienes y servicios a las comunidades históricamente afectadas por la presencia diferenciada del Estado y la incursión del conflicto armado interno. 
Un programa de articulación institucional con el apoyo de la empresa privada y cooperación internacional, que orienta sus esfuerzos al mejoramiento de las condiciones de vida de las poblaciones más vulnerables del territorio nacional, dentro del marco de la seguridad integral. (Ejército Nacional de Colombia, 2016, p. 3)

La implementación de este programa en Caquetá se dio en el 2016, como una iniciativa de la Décima Segunda Brigada del Ejército Nacional, con la finalidad de fortalecer la gobernabilidad mediante el empoderamiento de las comunidades. Para esto se promueven proyectos productivos, de infraestructura, mingas comunitarias, etc.

Es así como dicho programa busca:

[...] la articulación de las Instituciones del Estado, con el apoyo de la empresa privada y cooperación internacional que orienta sus esfuerzos al mejoramiento de las condiciones de vida de la población más vulnerable del Territorio Nacional, dentro del marco de la seguridad integral. (Ejército Nacional de Colombia, 2016, p. 3).

En otras palabras, generar una acción unificada del Estado en pro de municipios como Puerto Rico o San Vicente del Caguán, cuyas comunidades han sido altamente afectadas por el conflicto. Por ende, la pregunta ¿cuál es el rol del Ejército Nacional en la generación de desarrollo territorial?, es importante en tanto que, en tiempos de construcción de paz, los procesos de desarrollo requieren articulación de distintas instituciones. Además, la participación del Ejército Nacional en dichos procesos contribuye a saldar una deuda histórica de esta institución con el pueblo colombiano en su calidad de actor del conflicto armado.

Ahora bien, esta investigación es novedosa en tanto es un estudio de caso de un programa insigne de la acción integral reciente del Ejército Nacional; pero no es la única en esta materia. Por ejemplo, Adriana Lucía Cárdenas (2017) hace una investigación centrada en cómo el programa Fe en Colombia en Cauca contribuye a la consolidación del territorio. Pero, es con la investigación desarrollada por 
Alberto Castillo Castañeda y César Niño (2016) con la que más se relaciona el presente estudio, ya que dichos autores sostienen que la acción integral puede ser una política de seguridad en el posconflicto y aquí se analiza la forma en que el programa Fe en Colombia podría ser un ejemplo de cómo la acción integral del Ejército contribuye a la consecución de una seguridad ampliada en un escenario posterior al conflicto armado.

A su vez, se analiza el concepto de seguridad y de desarrollo que enmarca la acción integral del Ejército Nacional a través del programa en cuestión. En ese sentido, el punto de partida es que el Ejército Nacional es una institución que responde a una identidad y valores que son creados de manera intersubjetiva de acuerdo con el contexto.

\section{Marco TEÓRICO O CONCEPTUAL}

Para entender de qué manera el Ejército Nacional, a través de su programa Fe en Colombia, contribuye a la construcción de paz local, es preciso partir de la idea del Ejército como institución. De acuerdo con lo anterior, en este trabajo se entenderán a las instituciones como "las reglas del juego en una sociedad, o, fundamentalmente, son las restricciones humanamente entendidas que dan forma a las interacciones humanas y que en consecuencia estructuran los incentivos en el intercambio humano, ya sea político, social o económico" (North, 1990, citado en Vargas, 2005, p. 3). En otras palabras, el Ejército Nacional colombiano es una institución que, desde sus comienzos, ha sido determinante en la forma en que se relacionan los ciudadanos, definiendo las redes de cooperación.

Recordemos, además, que el institucionalismo sostiene que las instituciones permiten analizar y entender la realidad económica y política, en lugar de centrar el análisis en los individuos ya que, como se dijo, las instituciones constrińen los comportamientos de los individuos y mediante la acción colectiva actúan en representación del conglomerado social (Vargas, 2008). En ese orden de ideas, el Ejército Nacional es una institución a partir de la cual podríamos analizar la voluntad política y social de los caqueteños hacia la construcción de paz. 
Sin embargo, las instituciones no son estáticas, ni responden exclusivamente a las ideas e intereses de sus agentes, sino se transforman con base en el contexto, la coyuntura histórica y las dinámicas socioculturales. De ahí la importancia de incluir en nuestro análisis el enfoque constructivista, según el cual los intereses e identidades de las instituciones y del Estado mismo son construidos a partir de la interacción con otros actores (Wendt, 1999). En resumen:

El cambio organizacional está relacionado con el cambio de dichas reglas de juego que condicionan las formas de acceso al poder y las relaciones de los ciudadanos con los gobernantes. Los arreglos institucionales delimitan en forma contingente el juego político generando oportunidades, restricciones y riesgos al desarrollo. (Vargas, 2005, p. 4)

De acuerdo con lo anterior, el cambio organizacional y en las instituciones condiciona la forma en que se relacionan las personas y define el futuro político de las naciones, en tanto que se delimitan las formas de acceso al poder, relacionamiento entre gobernantes y ciudadanos.

Por otro lado, se tienen muy en cuenta las perspectivas de desarrollo, puesto que este es el principal propósito del programa Fe en Colombia: generar desarrollo en los territorios más afectados por el conflicto armado. No obstante, es preciso dilucidar qué entiende el Ejército Nacional por desarrollo y más si se tiene en cuenta que este:

Nunca ha sido un resultado espontáneo del mercado, sino que implica la acción de algunos actores sociales, la toma de decisiones políticas, la constitución de instituciones económicas y sociales específicas, el proceso que permita afianzar una identidad cultural propia, la energía social que haga posible despertar y dinamizar la creatividad. (Vidal y Guillén, 2007, p. 9)

De igual modo, el desarrollo no es el resultado del proceso de acumulación y crecimiento económico, sino que hace referencia a la expansión de las capacidades que pueden disfrutar las personas; es decir, es un proceso ligado al ser humano y su calidad de vida (Sen, 2000). Así lo entiende, a su vez, el Ejército Nacional de 
Colombia que, en el marco de su transformación hacia un Ejército del Futuro, se concibe que este "combatirá donde exista conflicto; no obstante, actuará como motor del desarrollo, contribuyendo al progreso, la calidad de vida, la preservación de la libertad y la garantía de los derechos" (Ciro y Correa, 2014, p. 10).

No es posible hablar de desarrollo sin hablar del concepto de seguridad, entendiendo esta desde su acepción amplia, conocida como seguridad humana, que ha sido definida por el Programa de Naciones Unidas para el Desarrollo (PNUD) en su informe de 1994 como "[un concepto que debe] estar centrado en las personas y no en el Estado, basado en las preocupaciones de la vida cotidiana de las personas y subrayando la importancia del desarrollo humano" (Cárdenas, 2017, p. 9).

En síntesis, se trata de analizar las acciones generadas en el marco del programa Fe en Colombia en el departamento del Caquetá, como iniciativas de desarrollo territorial lideradas por el Ejército Nacional en cumplimiento de sus nuevas funciones en el escenario de posconflicto.

\section{Metodología}

Se presenta un estudio de caso en la medida que busca:

Sistematiza(r) a lo largo de un período de tiempo una o varias experiencias o procesos, sus momentos críticos, actores y contexto con el fin de explorar sus causas, y entender por qué la/s experiencia/s o proceso/s objeto de estudio se desarrolló como lo hizo. (Rodríguez, 2011, p. 2).

Para ello se recurrió a fuentes de investigación cualitativa, como entrevistas semiestructuradas a dos profesionales del programa Fe en Colombia, que permitieron obtener información relacionada con el número de proyectos productivos y de infraestructura gestionados por la institución en los años 2017 y 2018, revisión de documentos oficiales del programa y observación participante. 
De igual manera, la metodología que se usó fue la descriptiva, ya que esta "tiene el propósito de definir las propiedades importantes de personas, grupos comunidades o cualquier otro fenómeno que sea sometido análisis, es decir, buscan saber 'quién', 'dónde', 'cuándo', 'cómo' y 'por qué' del sujeto de estudio” (Hernández, Fernández y Baptista, 1991, citado en Calderón, 2009, p. 52).

\section{ANTECEDENTES}

En el 2007, la III División del Ejército Nacional inicia un acompañamiento a proyectos productivos de comunidades indígenas y campesinas de Santander de Quilichao y municipios aledaños a Popayán, respectivamente, quienes sustituyeron sus cultivos de uso ilícito por cultivos de café, gracias a la articulación del Ejército Nacional, el Servicio Nacional de Aprendizaje (Sena), el Instituto Colombiano de Desarrollo Rural (Incoder), Acción Social de la Presidencia y algunos Ministerios (Ejército Nacional de Colombia, 2016b).

Debido al éxito obtenido en el acompañamiento del Ejército a las comunidades indígenas en proyectos productivos, en el 2008 la Tercera División creó en Popayán la Oficina de Asuntos Étnicos para generar un acercamiento con los asentamientos indígenas mediante el acompañamiento en proyectos productivos (Ejército Nacional de Colombia, 2016; Cárdenas, 2017). Luego:

El éxito en el departamento genera la necesidad expandir el programa y la oficina de asuntos étnicos evoluciona [en el 2012] a la oficina de proyectos productivos de la Tercera División, dependencia especializada en la difusión de la oferta estatal, rutas y articulación institucional. (Cárdenas, 2017, p. 23)

Mediante la Oficina de Proyectos Productivos se logró, en un año, gestionar 77 proyectos productivos que articularon los esfuerzos de instituciones como el Sena, el Ministerio de Agricultura, el Departamento de Prosperidad Social, el Incoder y algunas universidades. Con estas acciones se beneficiaron a más de 4000 personas con 
una inversión cercana a los 4 mil millones de pesos (Ejército Nacional de Colombia, 2016).

Tras el éxito rotundo del acompañamiento a comunidades indígenas en temas de proyectos, en el 2014 la Gobernación del Cauca, el Ejécito Nacional, las universidades y las alcaldías del departamento crearon un programa de articulación para acercar y ejecutar la política pública en las comunidades más vulnerables. Para ello se creó la campaña Fe en Colombia, que luego se convertiría en programa nacional con el objetivo ya mencionado (Ejército Nacional de Colombia, 2016; Cárdenas, 2017). Así, pues, Adriana Lucía Cárdenas (2017) explica que:

Para los propósitos respectivos se establecieron 3 líneas de trabajo; a. Líneas poblacionales, entendidas como aquellas en las que se generan espacios de acercamiento comunitario a campesinos, comunidades negras, indígenas y otras minorías; b. Líneas de acercamiento, especialmente diseńadas para abordar la dimensión económica de la seguridad, entendidas como aquellas herramientas de acción en las líneas poblacionales y que buscan generar oportunidades y polos de desarrollo en el territorio; (...) y iii. Líneas Transversales, a través de las cuales se transversalizan todos los procesos y se busca la cabal consolidación del territorio. (p. 24)

Una vez comprendido el surgimiento del programa Fe en Colombia, es preciso dilucidar el proceso de creación y ejecución de esta iniciativa en el departamento del Caquetá, para analizar, posteriormente, cuál es el papel del Ejército Nacional en la promoción de desarrollo territorial.

\section{Programa Fe en Colombia en el Caquetá}

En el transcurso del 2016, en la Décima Segunda Brigada del Ejército Nacional se inicia un diálogo con las agremiaciones y la academia del departamento del Caquetá, con el ánimo de articular esfuerzos entre los distintos sectores comprometidos por el desarrollo y mejoramiento de las condiciones de vida de los caqueteños. 
Fue así como el 20 de octubre de 2016 en Bogotá, en las instalaciones de la Escuela de Cadetes General José María Córdova, se lanzó el programa Fe en Colombia que, junto con el Departamento para la Prosperidad Social, la Cámara de Comercio de Florencia para el Caquetá, la Gobernación del Caquetá, la Universidad de la Amazonía, la Alcaldía de Florencia y gremios productivos del departamento, tenían como objetivo hacer del Caquetá un departamento próspero, impulsando el desarrollo. El evento contó con la presencia y participación de los agregados militares de Alemania, Argentina, Brasil, China, Chile, Ecuador, El Salvador, Honduras, Perú, Turquía, Corea, Italia, Francia, Rusia y Colombia, de manera que fuese posible focalizar inversiones y lograr que los diplomáticos multiplicasen la información a sus países de origen. Esto con el fin de mostrar al Caquetá como un destino turístico, seguro y con grandes propuestas e iniciativas en el sector empresarial (Ejército Nacional de Colombia, 2016c). De igual forma, el 17 de diciembre 2016 se realizó el lanzamiento de la iniciativa en la Plaza San Francisco en Florencia, el cual contó con la participación de diferentes agremiaciones del departamento.

Fe en Colombia es:

Un programa de articulación institucional con el apoyo de la empresa privada y cooperación internacional, que orienta sus esfuerzos al mejoramiento de las condiciones de vida de las poblaciones más vulnerables del territorio nacional, dentro del marco de la seguridad integral. (Ejército Nacional de Colombia, 2016a, p. 3)

Por lo tanto, constituye una estrategia de acción integral del Ejército Nacional.

El adecuado funcionamiento y éxito del programa depende no solo de la acción integral del Ejército Nacional, sino de la articulación entre las entidades e instituciones del Estado para solucionar las necesidades más apremiantes de las comunidades. Se trata, pues, del trabajo conjunto, combinado, coordinado e interagencial del sector estatal, el sector privado y las agencias de cooperación internacional. 


\section{Resultados}

Para determinar cuál es el aporte del Ejército Nacional de Colombia al desarrollo territorial en el Caquetá se analizaron los resultados del componente de proyectos productivos y de infraestructura gestionados desde el programa Fe en Colombia en el 2017 y 2018. Se hizo énfasis en las variables de número de proyectos, sus valores monetarios y sus beneficiarios, la diversidad de actores o cooperantes; $y$, de otro lado, la sistematización y centralidad de la información que se maneja.

\section{Número de proyectos}

Entre el 2017 y el 2018, el programa Fe en Colombia gestionó más de 15 proyectos productivos y más de 20 proyectos de infraestructura, con los que se logró impactar en las condiciones de vida de más de 15000 habitantes del Caquetá. Esto demuestra un incremento cuantitativo de los logros del programa, puesto que más del $50 \%$ de estas cifras se alcanzaron en el 2018 (figura 1).

Figura 1. Proyectos productivos y de infraestructura entre 2017-2018

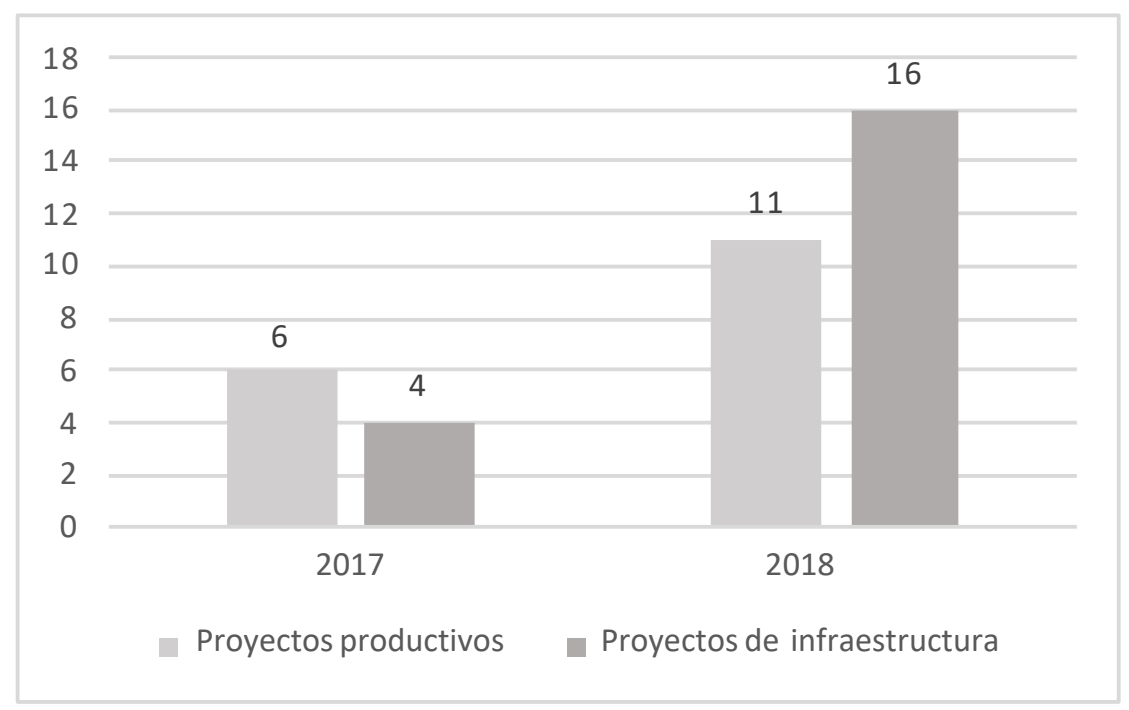

Fuente: elaboración propia. 
Sin embargo, llama la atención que los proyectos de infraestructura tuvieron una variación positiva en el 2018; es decir, si bien ambos sectores crecieron en el lapso estudiado, los proyectos productivos tuvieron un crecimiento estándar o constante, mientras que los de infraestructura pasaron de ser menos (frente a los productivos) en el 2017 a superar a los primeros en el 2018.

Lo anterior puede deberse al establecimiento de relaciones con aliados estratégicos que invirtieron recursos en mejorar la infraestructura vial del departamento como mecanismo para promover su productividad. Además, recordemos que el Ejército Nacional, en este caso la Décima Segunda Brigada, cuenta con un batallón de ingenieros, lo cual constituyó una gran ventaja, ya que el programa ofrece la mano de obra y algunas maquinarias como contrapartida en los proyectos de esta índole.

Ahora bien, los proyectos de infraestructura son una herramienta útil en la contribución del desarrollo de las comunidades más afectadas por el conflicto armado, ya que permiten reducir los costos de producción y comercialización de los productos de los campesinos (sobre todo agropecuarios); también facilitan el acceso a bienes y servicios del Estado, del sector privado y de cooperación internacional, de los cuales han sido históricamente rezagados. Es decir que el mejoramiento de vías, la construcción de centros de acopio, el restablecimiento de puentes, alcantarillados y demás acciones, inciden en la rentabilidad de iniciativas económicas de la región, y amplían las posibilidades de expandir las capacidades las personas a través del acceso y garantía a derechos como: salud y educación, atendiendo a un concepto de seguridad humana.

\section{Valor de los proyectos}

En esta variable la lógica cambia. Esto debido a que los proyectos productivos incrementaron su valor monetario del 2017 al 2018. Sin embargo, aun con dicha mejoría, el costo de estos ascendió máximo a 2500 millones de pesos colombianos, mientras que los proyectos de infraestructura tuvieron un crecimiento de 3000 a 5000 millones aproximadamente. En otras palabras, los proyectos de infraestructura presentaron un mayor crecimiento; incluso, en el 2017 cuando era superado en 
cantidad de proyectos por los productivos, su valor monetario era superior. Esto puede deberse a que los materiales y mano de obra de esta clase de proyectos suelen ser más costosos, porque responden a objetivos más ambiciosos y, además, en muchos casos, la infraestructura suele ser requisito sine qua non para el éxito de proyectos productivos (figura 2 ).

Figura 2. Valor monetario de los proyectos productivos y de infraestructura entre 2017-2018 (cop)

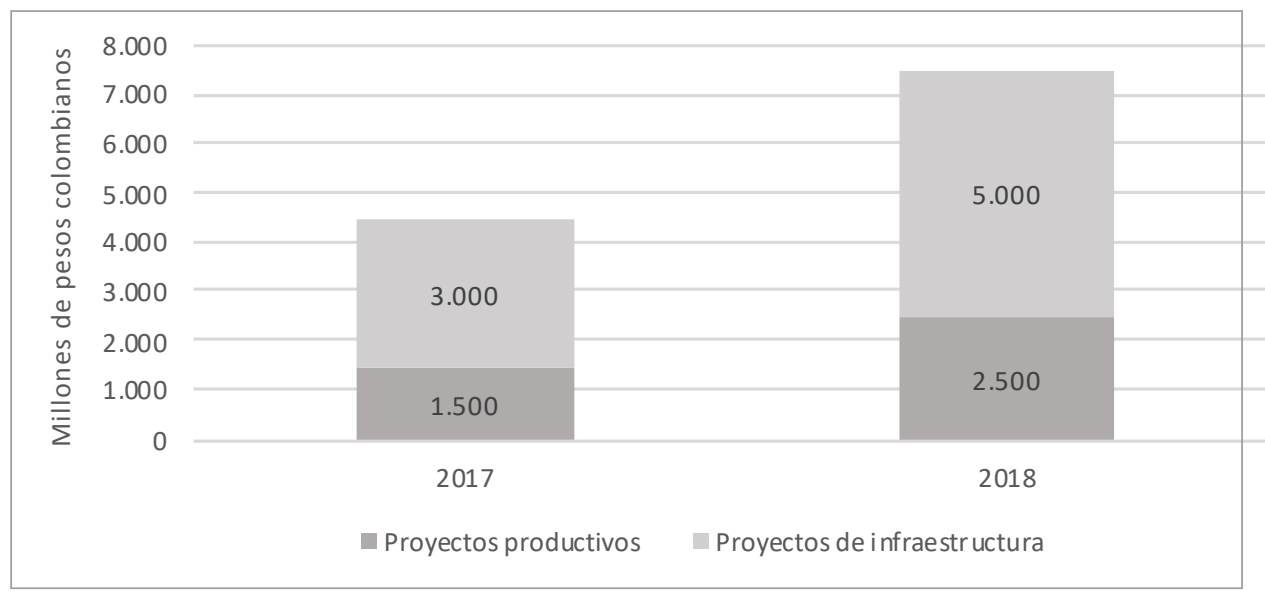

Fuente: elaboración propia.

\section{Número de beneficiarios}

Esta investigación pudo determinar que los proyectos productivos y de infraestructura gestionados por el programa $\mathrm{Fe}$ en Colombia han beneficiado a cerca de 15000 personas. El $33 \%$ de esta suma se alcanzó en el 2017 y el $67 \%$ restante se obtuvo mediante los proyectos del 2018 (figura 3).

Adicionalmente, en un artículo publicado en la revista de la aerolínea nacional EasyFly (2018), se relaciona que, hasta la fecha (diciembre 2018), 26 proyectos de infraestructura en 10 de los 16 municipios del Caquetá habían sido gestionados a través del programa, mientras que los proyectos productivos se localizaban en municipios como Puerto Rico, San Vicente del Caguán, Cartagena del Chairá, 
El Doncello, El Paujil, La Montańita, Puerto Milán y Solano (todos al norte del departamento); y San José del Fragua, como único municipio del sur del Caquetá, que fue beneficiado por Fe en Colombia con sus proyectos productivos.

Figura 3. Beneficiarios de los proyectos productivos y de infraestructura

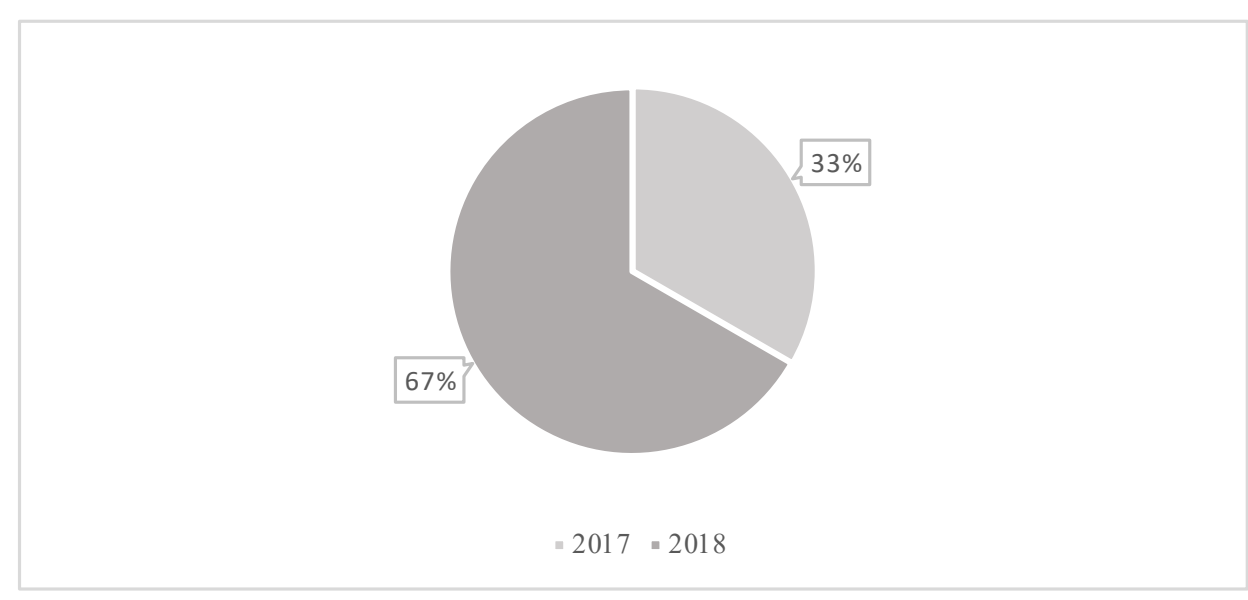

Fuente: elaboración propia.

Lo anterior pone sobre la mesa el debate acerca de los criterios de selección del programa para elegir las comunidades a impactar. Aunque las profesionales entrevistadas aseguraron que solo se tienen en cuenta tres requisitos: ser una comunidad organizada/asociada, no tener cultivos de uso ilícito y tener necesidades básicas insatisfechas, el análisis de los datos recogidos demuestra que existe una clara inclinación por impactar los municipios del norte del Caquetá que, entre otras cosas, son los más mediatizados por los efectos del conflicto armado. Por su parte, en el sur se ha optado por invisibilizar los rezagos de un conflicto que también se vivió, pero cuya historia parece no recordarlo.

Ahora, puede anotarse un quiebre interesante en el discurso de seguridad en el tema de posesión de cultivos de uso ilícito. Si bien el programa Fe en Colombia fue creado para generar desarrollo y bienestar en las comunidades más vulnerables a través de acciones interinstitucionales, uno de los criterios de selección para el 
apoyo en proyectos productivos y de infraestructura radica en la existencia de cultivos de uso ilícito en un departamento que, según la Oficina de las Naciones Unidas contra la Droga y el Delito (2019), para diciembre de 2018 ocupaba la sexta posición en número de hectáreas sembradas de coca en el país, pero paradójicamente es uno de los tres departamentos de Colombia que lidera la erradicación voluntaria verificadas por Naciones Unidas (Oficina de las Naciones Unidas contra la Droga y el Delito [unOdc], 2020). Es decir que el Ejército Nacional atiende el componente de sustitución de cultivos de uso ilícito desde un enfoque restringido de seguridad y no como una problemática social que es causa y efecto de las limitaciones a las capacidades de las poblaciones y sus libertades y que, por ende, les impide desarrollarse como quisieran.

Por lo anterior, cabe preguntarse si, de acuerdo con el objetivo del programa y en concordancia con la transformación hacia un Ejército del futuro, ¿̨no deberían ser las comunidades que se encuentran en proceso de erradicación y sustitución de cultivos de uso ilícito, una de las principales poblaciones beneficiarias y objeto del programa? $\mathrm{O}$, de cualquier forma, ¿la posesión de cultivos ilícitos no debería ser una condición excluyente para el acceso a los beneficios de Fe en Colombia?

Por último, el norte del departamento parece ofrecer una oportunidad histórica para el Ejército Nacional, en aras de saldar cuentas pendientes respecto al conflicto armado y ahora la construcción de paz, razón por la cual estos municipios reciben más atención y apoyo que la región del sur.

\section{Cooperantes}

De acuerdo con la información recolectada, en el 2017 el programa Fe en Colombia contó con ocho cooperantes o aliados estratégicos estables; es decir, que se mantuvieron en el 2018. Estos eran del orden nacional e institucional, por ejemplo, Invías y Prosperidad Social, e internacionales como Usaid, Turquía y la Unión Europea (tabla 1). 
Tabla 1. Mapa de actores cooperantes

\begin{tabular}{ll}
\hline $\begin{array}{c}\text { Cooperantes para proyectos productivos y } \\
\text { de infraestructura en el 2017 }\end{array}$ & $\begin{array}{c}\text { Cooperantes para proyectos productivos y de } \\
\text { infraestructura en el 2018 }\end{array}$ \\
\hline Agencia de Cooperación Turca Tika & Agencia de Cooperación Turca Tika \\
\hline Programa de Alianzas Comerciales de Usaid & Programa de Alianzas Comerciales de Usaid \\
\hline ACDI-voca Usaid & ACDI-voca Usaid \\
\hline Territorios de oportunidad de Usaid & Territorios de oportunidad de Usaid \\
\hline Ministerio de Defensa Nacional & Ministerio de Defensa Nacional \\
\hline Invías & Invías \\
\hline Unión Europea & Unión Europea \\
\hline Prosperidad Social & Prosperidad Social \\
\hline
\end{tabular}

Fuente: elaboración propia.

No obstante, hay dos aspectos que llamaron la atención de la información obtenida. En primer lugar, la constante presencia de Usaid y, por ende, de los Estados Unidos en el territorio, puesto que es el mismo cooperante a través de tres operadores y programas diferentes (ACDI-VOCA; Alianzas Comerciales y Territorios de Oportunidad), convirtiéndose así en un actor decisivo en la construcción de paz y generación de desarrollo territorial. En segundo lugar, es positivo que se haya logrado una continuidad de los mismos cooperantes en el 2018 en relación con el año anterior. Sin embargo, resulta necesario diversificar los actores o cooperantes, pues estos se van una vez cumplen su ciclo en un territorio; y en ese caso el programa se vería seriamente afectado, ya que está siendo dependiente de los mismos ocho actores.

Finalmente, Fe en Colombia es un programa que trabaja de la mano con las autoridades locales; es decir, no realiza esfuerzos de manera unilateral, sino que aspira a convertirse en el canalizador de la Ayuda Oficial al Desarrollo (AOD) que se direccione al departamento. Por esto, para el diseńo y ejecución de los proyectos se ha contado con el apoyo de las alcaldías municipales o Juntas de Acción Comunal (JAC). 


\section{Sistematización y centralidad de la información}

En relación con lo anterior, se identificó un problema estructural asociado con la alta vulnerabilidad del programa frente a los ocho cooperantes nombrados y es que, hasta el momento, en el departamento del Caquetá no existía una institución o área gubernamental que centralizara, organizara y dispusiera de la información relacionada con el número de cooperantes internacionales y donantes nacionales que hacen presencia en el territorio, por lo que la diversificación de los actores no es sencilla. Por otro lado, se generó desconocimiento respecto a los procesos, actores, recursos, beneficiarios, etc., de las iniciativas de desarrollo y construcción de paz.

En otras palabras, los actores internacionales no cumplen una ruta o puerta de ingreso en el departamento, sino que entran aleatoriamente. Esto dificulta para el programa Fe en Colombia el contacto con estos y la presentación y aplicación a sus convocatorias.

\section{¿Y estos proyectos sí generan desarrollo territorial?}

De acuerdo con las respuestas dadas por las dos profesionales entrevistadas, estos proyectos sí generaron desarrollo en las comunidades beneficiarias. No obstante, el discurso de la profesional 1 se diferenció sustancialmente de la terminología empleada por la profesional 2; pues, mientras que la primera asoció el desarrollo con la sostenibilidad de la economía de las familias beneficiadas, la segunda ligó el desarrollo con el empoderamiento de los campesinos y el fortalecimiento de su asociatividad —entendiendo esta como su participación en asociaciones formales y el mejoramiento de la calidad de vida-.

Podría decirse entonces que para la entrevistada 2 el Ejército Nacional, a través de este programa, brinda las herramientas necesarias para que los beneficiarios de los proyectos continúen por su propia cuenta con los procesos de desarrollo. Es decir, se trata de un empoderamiento, de la promoción de la asociatividad para que ellos mismos promuevan prácticas que les permitan mejoran su calidad de vida. Por otra parte, para la profesional 1 el desarrollo que está generando Ejército Nacional debe entenderse como el crecimiento económico de las familias. Las preguntas en este caso 
serían: ¿hasta cuándo ese crecimiento económico será sostenible?, ¿estas iniciativas del programa tienen entonces impacto a mediano o largo plazo? Además, esta concepción del desarrollo en términos económicos exclusivamente, no solo resulta reduccionista, sino que es desarticulada con la visión de desarrollo como mecanismo de expansión de las capacidades y libertades del ser humano (Sen, 2000).

En conclusión, la generación de proyectos productivos y de infraestructura desde el Programa Fe en Colombia constituye una transformación del Ejército Nacional de Colombia en un actor relevante para la construcción de paz y promoción del desarrollo en territorios afectados por el conflicto armado, como el Caquetá. Sin embargo, los proyectos por en sí mismos no generan desarrollo para las comunidades. No se trata solo de generar empleo o ingresos económicos, sino de satisfacer las necesidades básicas de las personas en materia de salud, educación, acceso a derechos y demás, las cuales les han sido negadas por las dinámicas propias de la violencia social y armada del país. Por otro lado, la sostenibilidad y continuidad de dichos proyectos es ampliamente discutida, ya que las condiciones políticas, económicas y sociales del departamento dificultan el éxito de iniciativas económicas distintas a la ganadería y cultivos de uso ilícito.

\section{Conclusiones}

El programa $\mathrm{Fe}$ en Colombia es una de las estrategias del Ejército Nacional, enmarcadas en su doctrina de acción integral, que se constituye como un mecanismo para desarrollar su acción social a través del fortalecimiento de las relaciones cívicomilitares y, en últimas, atender a la necesidad de transformación de la institución en un escenario de posconflicto. En el departamento del Caquetá este programa ha tenido gran acogida, especialmente por su componente de gestión y acompañamiento de proyectos productivos y de infraestructura para grupos poblaciones de víctimas, campesinos, indígenas, afrodescendientes y mujeres.

Entre el 2017 y el 2018, el programa Fe en Colombia gestionó más de 15 proyectos productivos y más de 20 proyectos de infraestructura, con los que se logró impactar 
en las condiciones de vida de más de 15000 habitantes del Caquetá. No obstante, estos fueron financiados por los mismos actores, lo que evidencia la necesidad de diversificar los cooperantes para que se reduzca el grado de vulnerabilidad del programa frente a estos.

Si bien el programa busca generar desarrollo atendiendo a un concepto amplio de seguridad (humana), a través de los proyectos productivos y de infraestructura, se evidencia una limitación en la aplicación de este, pues no es claro de qué manera dichas intervenciones contribuyen realmente al desarrollo (en un sentido amplio) del departamento en un mediano y largo plazo. Más allá de mejorar sus condiciones económicas momentáneamente, hace falta, entonces, fortalecer el componente de la oferta de bienes y servicios del Estado, y crear condiciones para la garantía de las libertades y la expansión de las capacidades de las personas.

Pese a ello, este programa se presenta como una ventana de oportunidad para que el Ejército Nacional amplíe sus funciones en el marco de un concepto ampliado de seguridad y en un contexto de posacuerdo. El Caquetá constituye un escenario idóneo para realizarlo, dada su historia política, social y económica marcada por el despojo, el conflicto, la violencia social y estructural en la que dicha institución ha ocupado un lugar preponderante.

\section{REFERENCIAS}

Calderón, C. (2009). Capítulo III. Definición de tipos de estudio. En P. Salas y M. Cárdenas (eds.), Métodos de investigación social (pp. 57-72). Ecuador: Editorial Ciespal.

Cárdenas, A. (2017). La Acción Integral como mecanismo de consolidación territorial. Revisión al programa Fe en Colombia en el departamento del Cauca (tesis inédita de especialización). Universidad Militar Nueva Granada, Granada, España. Recuperado de http://repository.unimilitar.edu.co/bitstream/10654/16899/3/CARDENASSUAREZ ADRIANALUCIA2017.pdf 
Castillo, A. y Niño, C. (2016). Doctrina de la acción integral como política de seguridad en el posconflicto armado en Colombia. En C. Niño (comp.), Perspectivas y prospectivas de la seguridad en Colombia (pp. 121-148). Bogotá: Universidad Santo Tomás.

Ciro, A. R. y Correa, M. (2014). Transformación estructural del Ejército colombiano. Construcción de escenarios futuros. Revista Cientifica General José Maria Córdova, 12(13), 19-88.

Departamento Nacional de Planeación. (2016). Indice de Incidencia del Conflicto Armado. Recuperado de http://colaboracion.dnp.gov.co/CDT/Poltica\%20de\%20Vctimas/ Construcci\%C3\%B3n\%20de\%20Paz/Documento\%20\%C3\%ADndice\%20de\%20 incidencia\%20del\%20conflicto\%20armado.pdf

EasyFly. (2018). Pasión Caquetá. Edición 109-diciembre 2018, pp. 38-45. Recuperado de https://issuu.com/revistaeasyfly/docs/revista_easyfly_109_isuue_1_

Ejército Nacional de Colombia. (2016a). Cartilla Fe en Colombia. Bogotá: Ejército Nacional de Colombia.

Ejército Nacional de Colombia. (2016b). Instructivo Fe en Colombia. Bogotá: Ejército Nacional de Colombia.

Ejército Nacional de Colombia. (2016c). Con éxito se hace lanzamiento de Pasión Caquetá. Recuperado de https://www.ejercito.mil.co/index.php/rss/recursos_user/ imagenes//?idcategoria $=407042$

Oficina de las Naciones Unidas contra la Droga y el Delito (UNODC). (2019). Colombia. Monitoreo de territorios afectados por cultivos ilícitos 2018. Recuperado de https://www. unodc.org/documents/colombia/2019/Agosto/Informe_de_Monitoreo_de_Territorios_Afectador_por_Cultivos_Ilicitos_en_Colombia_2018_.pdf

Oficina de las Naciones Unidas contra la Droga y el Delito (UNODC). (2020). Informe No. 19 Programa Nacional Integral de Sustitución de Cultivos de Uso Ilícitos - PNIS. Monitoreo a la 
implementación del Plan de Atención Inmediata. Recuperado de https://www.unodc.org/ documents/colombia/2020/Febrero/INFORME_EJECUTIVO_PNIS_No._19.pdf

Rodríguez, L. (2011). Pautas para la elaboración de casos de estudio. Sector de conocimiento y aprendizaje - Banco Interamericano de Desarrollo. Recuperado de https://publications. iadb.org/publications/spanish/document/Pautas-para-la-elaboraci\%C3\%B3n-de-estudios-de-caso.pdf

Sen, A. (2000). Desarrollo y Libertad. Buenos Aires: Editorial Planeta.

Vargas, J. (2005). Análisis de fundamentos del a teoría institucional. Revista Digital Universitaria, 6(8), 2-21. Recuperado de http://www.revista.unam.mx/vol.6/num8/ art84/ago_art84.pdf

Vargas, J. (2008). Perspectivas del Institucionalismo y Neoinstitucionalismo. Revista Ciencia Administrativa, 8, 47-58. Recuperado de https://www.uv.mx/iiesca/files/2012/12/perspectivas2008-1.pdf

Vidal, G. y Guillén, A. (2007). La necesidad de construir el desarrollo en América Latina. En G. Vidal y A. Guillén (comp.), Repensar la teoría del desarrollo en un contexto de globalización. Homenaje a Celso Furtado. Red de Bibliotecas Virtuales de Ciencias sociales de América Latina y el Caribe-Red Clacso. http://bibliotecavirtual.clacso.org.ar/ar/libros/ edicion/vidal_guillen/01Int ro.pdf

Wendt, A. (1999). Social Theory of International Politics. Cambridge: Cambridge UniversityPress. 